\title{
CORRESPONDENCE \\ Genomic landscape of hyperleukocytic acute myeloid leukemia
}

(c) The Author(s) 2021

Blood Cancer Journal (2022)12:4 ; https://doi.org/ 10.1038/s41408-021-00601-5

\section{Dear Editor,}

Approximately $20 \%$ of patients with acute myeloid leukemia (AML) present at diagnosis with hyperleukocytosis, which is commonly defined as a white blood cell count $>50 \times 10^{9} / \mathrm{L}$ or $>$ $100 \times 10^{9} / L$ [1]. Hyperleukocytic AML is an oncological emergency because the risk of early death is significant due to leukemic organ infiltration, leukostasis syndrome, disseminated intravascular coagulopathy and tumour lysis syndrome. Early management of these symptoms as well as rapid leukoreduction are critical in the therapeutic management [2].

In the era of next-generation sequencing (NGS), considerable progress has been made in understanding the genetic diversity of AML [3-5]. However, owing to the small proportion of hyperleukocytic patients generally included in clinical trials, the genomic landscape of hyperleukocytic AML and the prognostic impact of genetic lesions in this specific clinical context have not been described in detail except in a recent study from Taiwan which reported the frequency of mutations in a panel of 20 myeloid genes [6].

We recently reported the impact of dexamethasone in a series of 160 hyperleukocytic patients [7]. Here, we used this patient series to provide a molecular description of hyperleukocytic AML and to assess the prognostic impact of genetic classifications in patients treated with or without dexamethasone.

DEXAML-00 was a retrospective, single centre study comparing hyperleukocytic AML patients (18-75 years) who received intensive chemotherapy with $(n=60)$ or without $(n=100)$ dexamethasone between 2004 and 2015 [7]. Diagnostic samples for NGS analyses were available for 154 patients (96.3\%), 59 patients who received dexamethasone, and 95 patients who did not. Extended DNA resequencing was performed using an Illumina NextSeq500 and Sureselect (Agilent, Santa Clara, CA) targeted on the complete coding regions of 79 genes commonly mutated in myeloid malignancies (Supplementary data). Data were processed using two GATK algorithms, HaplotypeCaller (scaling accurate genetic variant discovery to tens of thousands of samples) and Mutect2, and via Agilent Surecall software, with a sensitivity of $1 \%$ $[8,9]$. All variants called by two variant callers, were checked using IGV software. Statistical analyses were performed using STATA software 14.2 (STATA Corp., College Station, TX).

Patient characteristics, results and outcome were unchanged compared to the first study (Table S1) [7]. Gene mutation frequency is shown in Fig. 1 and Table S2. The cytogenetic risk was favorable, intermediate or adverse in 15 (9.7\%), 121 (78.6\%) and $18(11.7 \%)$ patients, respectively. Genetic classifications, including the 2018 genomic classification, ELN 2017 risk classifier, NPM1/FLT3-ITD/DNMT3A mutational status and the functional gene categories, are shown in Table 1[3-5]. A total of 616 mutations were identified with an average of 4 mutations per patient (0-10 mutations/patient). Only one patient with inv(16) had no mutation. FLT3 (62.3\%), NPM1 (52.6\%), DNMT3A (34.4\%), TET2 (23.4\%), NRAS (20.8\%) were the most frequently mutated genes. Of the 71 patients (46\%) with FLT3-ITD mutations, 32 $(45.1 \%)$ had an allelic ratio $>0.5$. Mutations in the RAS pathway were detected in 67 patients (43.5\%), including NRAS (20.8\%), PTPN11 (9.7\%), KRAS (9.1\%), and NF1 (3.9\%). Overall, a large majority of patients had mutations in signaling genes $(n=131$, 85.1\%). Drug-actionable mutations such as FLT3 $(n=96), I D H 2$ $(n=17), I D H 1(n=14), K I T(n=5), \operatorname{TP} 53(n=4)$, or JAK2 $(n=1)$ were detected in 113 patients (73.4\%). In patients with FLT3 mutations, 12 had co-mutations in $I D H 1$, and 12 patients had comutations in $I D H 2$. Clinical characteristics and the distribution of actionable mutations according to main molecular subsets of the genomic classification and ELN 2017 are shown in Table S3 and S4.

We evaluated the prognostic impact of the genetic classifications as well as that of each individual gene according to the treatment group. In all multivariate survival analyses, no significant interaction between dexamethasone treatment and classifications or gene mutations was found, indicating that the effect of dexamethasone did not differ significantly between the various genetic subsets.

When the genomic classification was tested in multivariate analysis, AML with inv(16)/CBFB-MYH11 was the only subgroup with a significant impact on overall survival (OS) $(\mathrm{HR}, 0.16 ; 95 \% \mathrm{Cl}$ : $0.03-0.75 ; P=0.020$ ).

The co-occurrence of NPM1/FLT3-ITD/DNMT3A triple mutations has been shown to be associated with very poor OS.[5] This mutational status was observed in 25 patients (16\%), 23 of whom died. Compared to this triple mutated subset, lower HRs were found in double mutant NPM1mut/FLT3-ITD (HR, 0.43; 95\% Cl: $0.19-0.97 ; P=0.041)$ or NPM1mut/DNMT3Amut $(\mathrm{HR}, 0.47 ; 95 \% \mathrm{Cl}$ : $0.21-1.07 ; P=0.074)$.

Regarding functional gene categories, 2 subsets (NPM1mut: HR, 0.56; $95 \% \mathrm{Cl}: 0.33-0.97 ; P=0.039$ and myeloid transcription factor gene fusions or mutations: $\mathrm{HR}, 0.34 ; 95 \% \mathrm{Cl}: 0.19-0.60 ; P<0.001$ ) were significantly and independently associated with better OS whereas the chromatin-modifying gene subset was associated with poorer OS (HR, 1.88; 95\% Cl: $1.04-3.41 ; P=0.037$ ).

The ELN 2017 adverse group was independently associated with poor OS (HR, 2.53; 95\% Cl: $1.46-4.41 ; P=0.001)$. However there was no significant difference between intermediate and favorable prognostic groups (HR, 1.47; 95\% Cl: $0.87-2.48 ; P=$ 0.148 ).

Finally, we assessed the prognostic impact of each individual gene using the least absolute shrinkage and selection operator (LASSO) statistical method (Table S5). DNMT3A mutations were independently predictive of poor OS (HR, 1.76; $95 \% \mathrm{Cl}$ : 1.02-3.03; $P=0.043)$. On the contrary, CBFB-MYH11 (HR, $0.10 ; 95 \% \mathrm{Cl}$ : $0.02-0.43 ; P=0.002), C E B P A(\mathrm{HR}, 0.22 ; 95 \% \mathrm{Cl}: 0.09-0.53 ; P=$ $0.001), \quad N P M 1 \quad(\mathrm{HR}, 0.33 ; 95 \% \mathrm{Cl}: 0.19-0.58 ; P<0.001)$ and surprisingly, RUNX1 mutations (HR, $0.40 ; 95 \% \mathrm{Cl}$ : 0.18-0.92; $P=$ 0.030 ) were significantly and independently associated with better OS. The different types of RUNX1 mutations, co-mutations, 


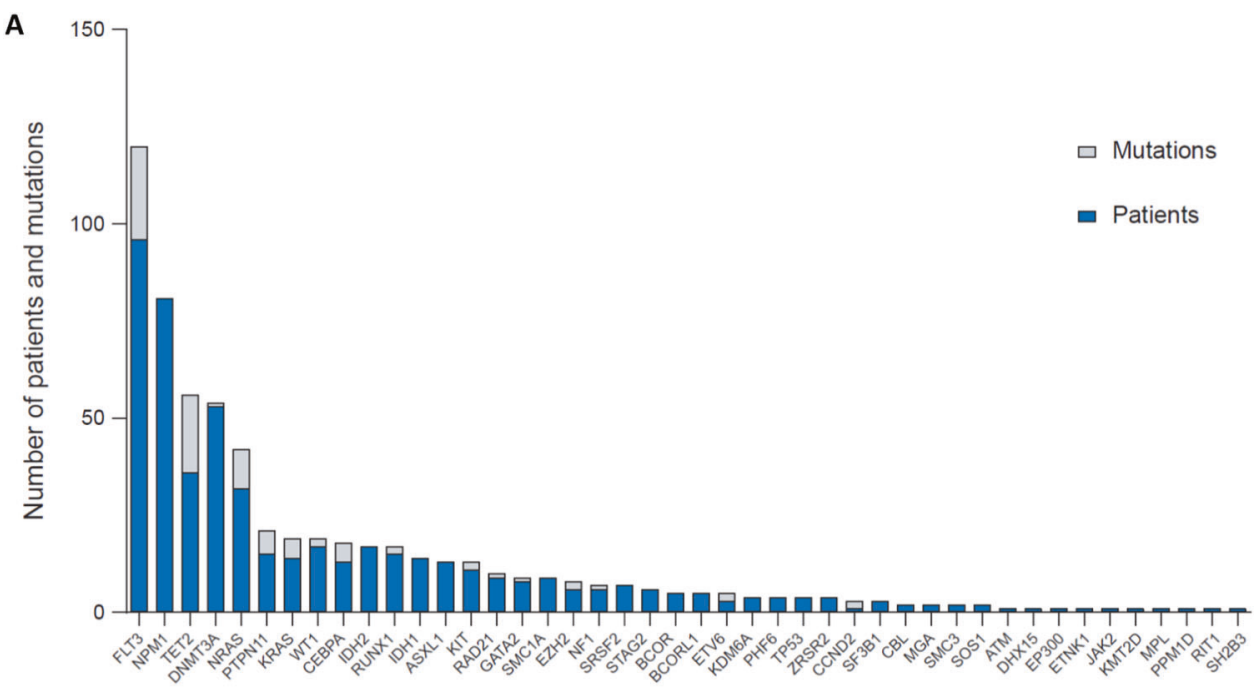

B

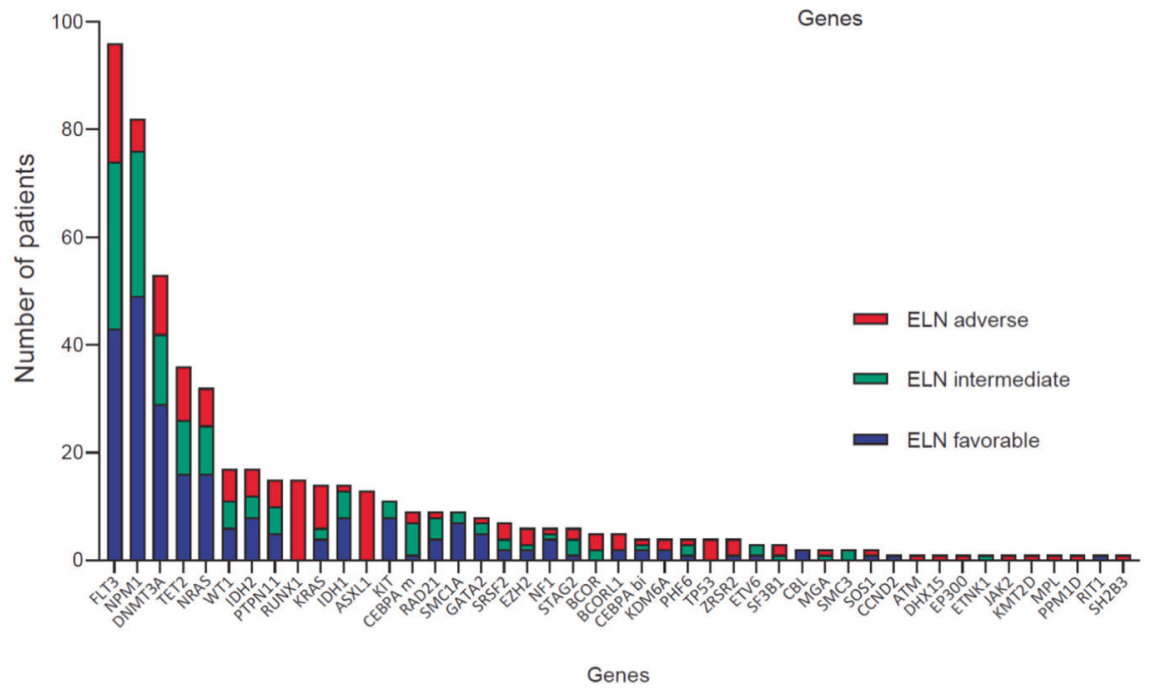

Fig. 1 Mutation pattern in hyperleukocytic AML. A Number of mutations (grey bars) and patients with mutations (blue bars) per gene. Of the 79 sequenced genes, 44 presented at least one mutation. B Distribution of mutations according to the 2017 European Leukaemia Net classification.

response to treatment with or without dexamethasone and outcome are shown in Table S6. Of the 15 patients with $R U N X 1^{\text {mut }}$ AML, 13 (86.7\%) achieved a complete response.

Multivariate analyses for event-free survival, relapse-free survival and cumulative incidence of relapse yielded similar results (data not shown). Of note, clinical or treatment parameters including infection at diagnosis, secondary $\mathrm{AML}$, hydroxyurea, albumin, LDH, fibrinogen, CD56 expression, admission to intensive care unit (ICU) or allogeneic stem-cell transplantation retained an independent prognostic value in most multivariate analyses (Table S5).

This study shows that the genomics of hyperleukocytic AML differs substantially from nonhyperleukocytic AML. Signalling mutations in either FLT3 or RAS pathways, mutations in DNA methylation genes and NPM1 were over-represented whereas other subgroups, such as RUNX1-RUNX1T1 or TP53 mutations had a very low frequency. Compared to the Taiwanese study, we found far fewer CEBPA biallelic mutations (2.6\% vs. $16 \%$ ) and more NPM1 (53.2\% vs. $30 \%$ ) or FLT3 mutations (62.3\% vs. $44.5 \%)$. Apart from the difference in median age between the two cohorts (60 vs. $50 \mathrm{y}$ ), we have no clear explanation for this difference [6].

A large proportion of hyperleukocytic AML patients present at diagnosis with therapeutically targetable mutations (>70\%).
This brings hope that the use of targeted therapies may improve their prognosis, which is still poor compared to nonhyperleukocytic AML. Midostaurin combined with intensive chemotherapy has improved the outcome of AML patients with FTL3 mutations ( $60 \%$ of patients in our series) including ELN 2017 adverse risk patients [10]. Our patients were treated before midostaurin approval in Europe and therefore no patient received a FLT3 inhibitor. Thus, further studies are needed to assess the impact of midostaurin in hyperleukocytic AML. Inhibition of the RAS pathway could also be a valuable avenue in this setting [11].

AML with $R U N X 1$ mutations ( $R U N X 1^{\text {mut }} \mathrm{AML}$ ) is a provisional entity of the WHO 2016 classification that accounts for $10 \%$ of newly diagnosed AML. In our series of hyperleukocytic patients, the frequency of $R U N X 1$ mutations was similar ( $n=15,9.7 \%)$. RUNX $1^{\text {mut }}$ AML are classified in the ELN 2017 adverse risk group. However, RUNX1 mutations were independently associated with better OS in our cohort. A recent study using a chemogenomic approach revealed that AML cells with inactivating RUNX1 mutations were particularly sensitive to very low concentrations of glucocorticoids [12]. However, given the small patient cohort, we were unable to link this improved outcome to dexamethasone treatment-hence the clinical impact of glucocorticoids in $R U N X 1^{\text {mut }} \mathrm{AML}$ has yet to be determined. 
Table 1. Genetic Classifications of Hyperleukocytic AML.

\begin{tabular}{|c|c|c|c|c|}
\hline & \multicolumn{2}{|c|}{ Dexamethasone } & \multirow[t]{2}{*}{$P$ value } & \multirow{2}{*}{$\begin{array}{l}\text { Total } \\
154(100.0)\end{array}$} \\
\hline & $\begin{array}{l}\text { No } \\
95(\%)(61.7)\end{array}$ & $\begin{array}{l}\text { Yes } \\
59(\%)(38.3)\end{array}$ & & \\
\hline Genomic classification* - $n(\%)$ & & & 0.176 & \\
\hline AML with NPM1 mutation & $48(50.5)$ & $32(54.2)$ & & $80(51.9)$ \\
\hline AML with mutated chromatin, RNA-splicing genes, or both & $22(23.2)$ & 7 (11.9) & & $29(18.8)$ \\
\hline AML with TP53 mutations, chromosomal aneuploidy, or both & $4(4.2)$ & $0(0.0)$ & & $4(2.6)$ \\
\hline AML with $\mathrm{t}(8 ; 21)(\mathrm{q} 22 ; \mathrm{q} 22) ; \mathrm{RUNX1-RUNX1T1}$ & $2(2.1)$ & $0(0.0)$ & & $2(1.3)$ \\
\hline AML with MLL fusion genes; $t(x ; 11)(x ; q 23)$ & $4(4.2)$ & $2(3.4)$ & & $6(3.9)$ \\
\hline AML with IDH2R172 mutations and no other class-defining lesions & $0(0.0)$ & $1(1.7)$ & & $1(0.6)$ \\
\hline ELN $2017-n(\%)$ & & & 0.231 & \\
\hline Favorable & $36(37.9)$ & $30(50.8)$ & & $66(42.9)$ \\
\hline $\mathrm{NPM} 1=1, \mathrm{FLT3}-\mathrm{ITD}=1, \mathrm{DNMT} 3 \mathrm{~A}=0$ & $10(10.5)$ & $14(23.7)$ & & $24(15.6)$ \\
\hline $\mathrm{NPM} 1=1, \mathrm{FLT3}-\mathrm{ITD}=0, \mathrm{DNMT} 3 \mathrm{~A}=1$ & $12(12.6)$ & $6(10.2)$ & & $18(11.7)$ \\
\hline $\mathrm{NPM} 1=1, \mathrm{FLT3}-\mathrm{ITD}=0, \mathrm{DNMT} 3 \mathrm{~A}=0$ & $9(9.5)$ & $6(10.2)$ & & $15(9.7)$ \\
\hline $\mathrm{NPM} 1=0, \mathrm{FLT3}-\mathrm{ITD}=1, \mathrm{DNMT} 3 \mathrm{~A}=1$ & $5(5.3)$ & $1(1.7)$ & & $6(3.9)$ \\
\hline$N P M 1=0, F L T 3-I T D=1$, DNMT3A $=0$ & $8(8.4)$ & $8(13.6)$ & & $16(10.4)$ \\
\hline $\mathrm{NPM} 1=0, \mathrm{FLT3}-\mathrm{ITD}=0, \mathrm{DNMT} 3 \mathrm{~A}=1$ & $2(2.1)$ & $2(3.4)$ & & $4(2.6)$ \\
\hline$N P M 1=0, F L T 3-I T D=0, D N M T 3 A=0$ & $31(32.6)$ & $15(25.4)$ & & $46(29.9)$ \\
\hline \multicolumn{5}{|l|}{ Functional categories - $\boldsymbol{n}(\%)$} \\
\hline \multicolumn{5}{|l|}{ NPM1 mutation } \\
\hline No & $46(48.4)$ & $26(44.1)$ & & $72(46.8)$ \\
\hline Yes & 49 (51.6) & $33(55.9)$ & 0.598 & $82(53.2)$ \\
\hline Yes & $81(85.3)$ & $50(84.7)$ & 0.930 & $131(85.1)$ \\
\hline \multicolumn{5}{|l|}{ Myeloid TF gene fusions or mutations } \\
\hline No & $64(67.4)$ & $37(62.7)$ & & $101(65.6)$ \\
\hline Yes & $31(32.6)$ & $22(37.3)$ & 0.554 & $53(34.4)$ \\
\hline \multicolumn{5}{|l|}{ Chromatin-modifying genes } \\
\hline No & $77(81.1)$ & $50(84.7)$ & & $127(82.5)$ \\
\hline Yes & $18(18.9)$ & $9(15.3)$ & 0.557 & $27(17.5)$ \\
\hline \multicolumn{5}{|l|}{ Cohesin-complex genes } \\
\hline No & $77(81.1)$ & $50(84.7)$ & & $127(82.5)$ \\
\hline Yes & $18(18.9)$ & $9(15.3)$ & 0.557 & $27(17.5)$ \\
\hline \multicolumn{5}{|l|}{ Spliceosome-complex genes } \\
\hline No & 85 (89.5) & $54(91.5)$ & & $139(90.3)$ \\
\hline Yes & $10(10.5)$ & $5(8.5)$ & 0.676 & $15(9.7)$ \\
\hline \multicolumn{5}{|l|}{ Others } \\
\hline No & $88(92.6)$ & 57 (96.6) & & $145(94.2)$ \\
\hline Yes & $7(7.4)$ & $2(3.4)$ & 0.483 & $9(5.8)$ \\
\hline
\end{tabular}

*Compared to the genomic classification, AML with $\mathrm{t}(15 ; 17)(\mathrm{q} 22 ; \mathrm{q} 12) ; \mathrm{PML}-\mathrm{RARA}$ were excluded, whereas some subsets including AML with inv(3)(q21q26.2) or $\mathrm{t}(3 ; 3)(\mathrm{q} 21 ; \mathrm{q} 26.2)$; GATA2, MECOM(EVI1), AML with $\mathrm{t}(6 ; 9)(\mathrm{p} 23 ; \mathrm{q} 34)$; DEK-NUP214, AML with no detected driver mutations, AML meeting criteria for $\geq 2$ genomic subgroups were not found in hyperleukocytic AML.

ELN, European Leukemia Net. 
Studies on the mechanisms of resistance to chemotherapy or tyrosine kinase inhibitors together with high-throughput drug screening have underpinned the potential role of glucocorticoids in AML. Cytarabine resistance is associated with the acquisition of sensitivity to glucocorticoids and mutated RUNX1, NPM1, or SRSF2 modulate gene expression in a manner that primes AML cells for glucocorticoid sensitivity [13]. These recent data suggest that some AML subgroups (not necessarily hyperleukocytic patients) may specifically benefit from dexamethasone. However, we did not find any significant interaction between dexamethasone treatment and genomic alterations. This may be due to insufficient numbers or dexamethasone may have broader effects on biological phenomena such as inflammation, microenvironment or leukemic stem-cell biology.

Hyperleukocytic AML remains a very challenging clinical management issue. This is evidenced by the high number of multivariate analysis parameters that reflect the patients' general condition, inflammation, metabolism or therapeutic management. Acting on these parameters could be key to limiting early complications and induction deaths. Leukoreduction with hydroxyurea, anti-inflammatory treatments, such as dexamethasone and early or direct admission to ICU have recently been proposed to this end whereas the benefit of leukapheresis has not been demonstrated [14]. New targeted therapies that may cover the majority of hyperleukocytic patients as shown in our study and a better understanding of the mechanisms of leukostasis could help to improve patient management and prognosis [15].

Laetitia Largeaud ${ }^{1,2,3,8}$, Sarah Bertoli (iD ${ }^{2,3,4,8}$, Emilie Bérard ${ }^{5,6,8}$, Suzanne Tavitian ${ }^{4}$, Muriel Picard ${ }^{7}$, Stéphanie Dufrechou', Naïs Prade ${ }^{1}$, François Vergez ${ }^{1,2,3}$, Jean Baptiste Rieu (D) ${ }^{1}$, Isabelle Luquet ${ }^{1}$, Audrey Sarry ${ }^{4}$, Françoise Huguet ${ }^{4}$, Jean Ruiz ${ }^{7}$, Véronique De Mas (iD ${ }^{1,2,3}$ Eric Delabesse ${ }^{1,2,3,8}$ and Christian Récher (iD ${ }^{2,3,4,8}{ }^{\bowtie \prime}$

${ }^{1}$ Centre Hospitalier Universitaire de Toulouse, Institut Universitaire du Cancer de Toulouse Oncopole, Laboratoire d'Hématologie, Toulouse, France. ${ }^{2}$ Université Toulouse III Paul Sabatier, Toulouse, France.

${ }^{3}$ Cancer Research Center of Toulouse, UMR1037-INSERM, ERL5294 CNRS Toulouse, France. ${ }^{4}$ Centre Hospitalier Universitaire de Toulouse, Institut Universitaire du Cancer de Toulouse Oncopole, Service d'Hématologie, Toulouse, France. ${ }^{5}$ Centre Hospitalier Universitaire de Toulouse, Service d'Epidémiologie, Toulouse, France. ${ }^{6}$ UMR 1295

CERPOP, INSERM-Université de Toulouse III, Toulouse, France. ${ }^{7}$ Service de Réanimation Polyvalente, Centre Hospitalier Universitaire de Toulouse, Institut Universitaire du Cancer de Toulouse Oncopole, Toulouse, France. ${ }^{8}$ These authors contributed equally: Laetitia Largeaud, Sarah Bertoli, Emilie Bérard, Eric Delabesse, Christian Récher. ${ }^{\bowtie}$ email: recher.christian@iuct-oncopole.fr

\section{REFERENCES}

1. Rollig C, Ehninger G. How I treat hyperleukocytosis in acute myeloid leukemia. Blood 2015;125:3246-52.

2. Stahl M, Shallis RM, Wei W, Montesinos P, Lengline E, Neukirchen J, et al. Management of hyperleukocytosis and impact of leukapheresis among patients with acute myeloid leukemia (AML) on short- and long-term clinical outcomes: a large, retrospective, multicenter, international study. Leukemia 2020;34:3149-60.

3. Dohner H, Estey E, Grimwade D, Amadori S, Appelbaum FR, Buchner T, et al. Diagnosis and management of AML in adults: 2017 ELN recommendations from an international expert panel. Blood 2017;129:424-47.

4. Bullinger L, Dohner K, Dohner H. Genomics of Acute Myeloid Leukemia Diagnosis and Pathways. J Clin Oncol. 2017;35:934-46.

5. Papaemmanuil E, Gerstung M, Bullinger L, Gaidzik VI, Paschka P, Roberts ND, et al. Genomic Classification and Prognosis in Acute Myeloid Leukemia. N. Engl J Med. 2016;374:2209-21.

6. Tien FM, Hou HA, Tsai CH, Tang JL, Chen CY, Kuo YY, et al. Hyperleukocytosis is associated with distinct genetic alterations and is an independent poor-risk factor in de novo acute myeloid leukemia patients. Eur J Haematol. 2018;101:86-94.
7. Bertoli S, Picard M, Berard E, Griessinger E, Larrue C, Mouchel PL, et al. Dexamethasone in hyperleukocytic acute myeloid leukemia. Haematologica 2018;103:988-98.

8. McKenna A, Hanna M, Banks E, Sivachenko A, Cibulskis K, Kernytsky A, et al. The Genome Analysis Toolkit: a MapReduce framework for analyzing next-generation DNA sequencing data. Genome Res. 2010;20:1297-303.

9. Poplin R, Ruano-Rubio V, DePristo MA, Fennell TJ, Carneiro MO, Van der Auwera $\mathrm{GA}$, et al. Scaling accurate genetic variant discovery to tens of thousands of samples. bioRxiv. 2018:201178.

10. Döhner K, Thiede C, Jahn N, Panina E, Gambietz A, Larson RA, et al. Impact of NPM1/FLT3-ITD genotypes defined by the 2017 European LeukemiaNet in patients with acute myeloid leukemia. Blood 2020;135:371-80.

11. Tambe M, Karjalainen E, Vähä-Koskela M, Bulanova D, Gjertsen BT, Kontro $M$, et al. Pan-RAF inhibition induces apoptosis in acute myeloid leukemia cells and synergizes with BCL2 inhibition. Leukemia 2020;34:3186-96.

12. Simon L, Lavallée VP, Bordeleau ME, Krosl J, Baccelli I, Boucher G, et al. Chemogenomic Landscape of RUNX1-mutated AML Reveals Importance of RUNX1 Allele Dosage in Genetics and Glucocorticoid Sensitivity. Clin Cancer Res. 2017;23: 6969-81.

13. Récher C. Clinical Implications of Inflammation in Acute Myeloid Leukemia. Front Oncol. 2021;11:623952.

14. Saillard C, Elkaim E, Rey J, d'Incan E, Charbonnier A, Etienne A, et al. Early preemptive ICU admission for newly diagnosed high-risk acute myeloid leukemia patients. Leuk Res. 2018;68:29-31.

15. Bewersdorf JP, Zeidan AM. Hyperleukocytosis and Leukostasis in Acute Myeloid Leukemia: Can a Better Understanding of the Underlying Molecular Pathophysiology Lead to Novel Treatments? Cells. 2020;9:2310.

\section{ACKNOWLEDGEMENTS}

We would like to thank the Data Management Unit of Toulouse University Hospital. We are also very grateful to all members of the G.A.E.L (Gaël Adolescent Espoir Leucémie) Association. This work has been grant-funded by the $\mathrm{CHU}$ de Toulouse (local grant 2018), the Toulouse Cancer Santé Foundation (Toulouse Cancer Health Foundation), the FONROGA Foundation, and the Ligue Contre le Cancer (Anti-Cancer League) Foundation (AO 2018).

\section{AUTHOR CONTRIBUTIONS}

L.L., S.D., N.P., V.D.M., and E.D. performed molecular analyses; L.L., E.D. interpreted molecular data; E.B. performed statistical analysis and wrote the paper; S.B., S.T., M.P., F.H., J.R. collected clinical data and/or treated patients; AS collected data; F.V., J.B.R., I.L. performed initial AML work-up; C.R. collected data, treated patients, supervised analysis and wrote the paper. The manuscript was prepared by all authors with the assistance of a medical writer funded by the CHU Toulouse. All authors reviewed, provided comments and approved the manuscript.

\section{COMPETING INTERESTS}

Christian Récher: Research grants (my institution): Abbvie, Amgen, Novartis, BMSCelgene, Jazz Pharma, Agios, Daiichi-Sankyo, Astellas, Roche, MaatPharma, Iqvia. Advisory boards: Abbvie, Janssen, Jazz Pharma, Daiichi-Sankyo, Astellas, Novartis, Celgene, Takeda, Roche, Otsuka, Macrogenics, Pfizer; Sarah Bertoli: advisory role for Jazz Pharmaceuticals, Daiichi-Sankyo, Sanofi, Astellas and BMS; François Vergez: research grants from Pierre Fabre and Roche; is an advisor for Astellas and Amgen; Françoise Huguet: consultancy Novartis, Pfizer, Incyte; honoraria Amgen, Servier; Isabelle Luquet: advisory role for Jazz Pharmaceuticals.

\section{ADDITIONAL INFORMATION}

Supplementary information The online version contains supplementary material available at https://doi.org/10.1038/s41408-021-00601-5.

Correspondence and requests for materials should be addressed to Christian Récher.

Reprints and permission information is available at http://www.nature.com/ reprints

Publisher's note Springer Nature remains neutral with regard to jurisdictional claims in published maps and institutional affiliations. 
Open Access This article is licensed under a Creative Commons At c) Atribution 4.0 International License, which permits use, sharing,
adaptation, distribution and reproduction in any medium or format, as long as you give appropriate credit to the original author(s) and the source, provide a link to the Creative Commons license, and indicate if changes were made. The images or other third party material in this article are included in the article's Creative Commons license, unless indicated otherwise in a credit line to the material. If material is not included in the article's Creative Commons license and your intended use is not permitted by statutory regulation or exceeds the permitted use, you will need to obtain permission directly from the copyright holder. To view a copy of this license, visit http://creativecommons. org/licenses/by/4.0/.

(c) The Author(s) 2021 